\title{
Efeito de um período de polimento sobre o estado de humor de nadadores
}

http://dx.doi.org/10.11606/1807-5509201700030535

\author{
Leonardo de Sousa FORTES* \\ Maria Elisa Caputo FERREIRA** \\ Saulo Fernandes Melo de OLIVEIRA* \\ Lenamar Fiorese VIEIRA*** \\ *Núcleo de Educação \\ Física e Ciências \\ do Desporto, \\ Universidade Federal \\ de Pernambuco, \\ Recife, PE, Brasil. \\ **Faculdade de \\ Educação Física \\ e Desportos, \\ Universidade Federal \\ de Juiz de Fora, Juiz \\ de Fora, MG, Brasil. \\ ***Departamento de \\ Educação Física, \\ Universidade Estadual \\ de Maringá, Maringá, \\ PR, Brasil.
}

\section{Resumo}

Parece haver relação positiva entre o estado de humor e o desempenho esportivo. Todavia, embora pesquisadores ressaltem que o polimento pode acarretar melhora do humor, cabe destacar que tal adaptação necessita ser confirmada a partir de achados científicos. Pouco se sabe a respeito do efeito do polimento sobre o estado de humor de atletas. 0 objetivo foi analisar o efeito do polimento sobre o estado de humor de nadadores. Participaram 62 atletas de natação do sexo masculino, divididos aleatoriamente em grupo experimental (GE) e controle (GC). Ambos os grupos fizeram a mesma planificação de treinamento até a fase do polimento. Somente o GE realizou o polimento. 0 polimento teve duração de 3 semanas, adotando-se o método de polimento linear. A Escala de Humor de Brunel (BRUMS) foi preenchida pelos atletas antes do início da temporada e na última semana de cada mesociclo. Conduziu-se a análise multivariada de covariância (MANOVA) de medidas repetidas para comparar as subescalas da BRUMS entre os grupos em função do mesociclo. Os resultados apresentaram efeitos de tempo $\left(F_{(4,58)}=34,02, p=0,001\right)$ e grupo $\left(F_{(2,60)}=28,91, p=0,001\right)$. De maneira geral, os achados do presente estudo revelaram o aumento do chamado perfil "iceberg" após o polimento para o GE. Concluiu-se que o polimento foi eficaz para melhorar o estado de humor de nadadores, com destaque para o aumento do vigor e diminuição da raiva, fadiga e depressão.

Palavras-chave: Psicologia do Esporte; Humor; Atletas.

\section{Introdução}

Sessôes de treinamento físico, tático, técnico e psicológico fazem parte da rotina diária de atletas ${ }^{1}$. O planejamento do treinamento de atletas é fundamental para se maximizar o desempenho esportivo. Este planejamento é conhecido como periodização do treinamento ${ }^{2}$. Mais especificamente, nos esportes cíclicos, tais como ciclismo, remo e natação, treinadores costumam utilizar a periodização simples ou dupla ${ }^{3}$.

Na periodização simples o treinador busca planejar as cargas de treinamento com o intuito de que seus atletas atinjam o melhor desempenho em uma única competição ao final do macrociclo ${ }^{4}$. Na natação este tipo de periodização é composta por quatro etapas, a saber: preparatório geral, preparatório específico, competitivo e polimento ${ }^{5}$. As duas primeiras fases são utilizadas para se aumentar progressivamente volume e intensidade do treinamento. No período competitivo é aumentado a frequência de realização de séries de alta intensidade ao mesmo tempo que o volume de treinamento aeróbio é reduzido. $\mathrm{O}$ polimento, por sua vez, é utilizado para se reduzir 
o volume de todos os componentes das sessóes de treino, embora a intensidade seja mantida ${ }^{6}$. Estudos têm indicado aumento do desempenho de nadadores após a etapa do polimento ${ }^{3,7}$. No entanto, a prescriçáo do polimento parece náo ser unanimidade para muitos treinadores ${ }^{2}$. Ainda paira uma subcultura de que nas semanas antecedentes a competição-alvo tanto volume quanto intensidade devem ser aumentados.

Parece que as adaptaçóes psicológicas (motivação, autoeficácia e estado de humor) oriundas do polimento ainda não são claras. Embora MujıKa et al. $^{7}$ ressaltem que o polimento pode acarretar aumento da motivação, autoeficácia, melhora do humor e redução do estresse mental, cabe destacar que tais adaptaçóes necessitam ser confirmadas a partir de achados científicos.

$\mathrm{O}$ estado de humor refere-se ao conjunto de sentimentos positivos e negativos que variam em intensidade e duração ${ }^{8}$. Fazem parte do estado de humor: depressão, tensão, raiva, fadiga, confusão mental e vigor. Estudos têm revelado relação positiva entre o estado de humor com perfil "iceberg" (vigor elevado e os demais sentimentos reduzidos) e o desempenho esportivo?. De acordo com Rouverx et al..$^{9}$ o estado de humor costuma ser alterado em razão das cargas de treinamento físico (volume e intensidade). Todavia, não sabemos se o polimento (caracterizado pela atenuaçáo do volume de treinamento) é capaz de gerar adaptações positivas no estado de humor. Do ponto de vista prático, este tipo de investigação contribuiria para os treinadores terem conhecimento concernente as adaptaçôes psicológicas que a fase do polimento pode gerar em seus atletas. Em virtude dos apontamentos supracitados, o objetivo do estudo foi analisar o efeito do polimento sobre o estado de humor de nadadores.

Por conseguinte, duas hipóteses foram formuladas, baseando-se nos apontamentos de duas revisóes sistemáticas ${ }^{4,6}:$ a) o polimento gera aumento da sensação de vigor e; b) a fase do polimento reduz a magnitude do humor negativo (depressão, tensão, raiva, fadiga e confusáo mental).

\section{Método}

\section{Participantes}

Trata-se de uma investigação experimental com follow-up de 20 semanas desenvolvida com nadadores do sexo masculino. A amostra foi composta por 70 voluntários com idade entre 15 e 17 anos, participantes do campeonato mineiro de natação das categorias juvenil ou júnior. Os participantes foram divididos aleatoriamente em dois grupos: grupo experimental $(\mathrm{GE}, \mathrm{n}=35)$ e grupo controle $(\mathrm{GC}, \mathrm{n}=35)$. Salienta-se que não se identificaram diferenças estatísticas para idade $\left(F_{(2,68)}=3,01, p=0,19\right)$, percentual de gordura $\left(F_{(2,68)}=2,56, p=0,23\right)$ e estado de humor $\left(F_{(2,68)}=1,95, p=0,27\right)$ entre GE e GC antes do início da investigação.

Os nadadores treinavam em média $2 \mathrm{~h}$ por dia, com frequência de cinco vezes por semana. Para serem incluídos na pesquisa, os atletas deveriam: a) ser atleta de natação a pelo menos dois anos; b) treinar sistematicamente natação por pelo menos 8 h por semana; e c) ter índice para o campeonato estadual (Minas Gerais), organizado pela Federação Aquática Mineira.
Contudo, 8 atletas foram excluídos em razão de faltarem mais do que $5 \%$ das sessóes de treinamento no decorrer da investigação (20 semanas) ou apresentarem questionário incompleto em uma das etapas da pesquisa. Logo, a investigação contou com uma amostra final de 62 nadadores ( $\mathrm{GE}=30$ e $\mathrm{GC}=32$ ). Ressalta-se que após a exclusão dos 8 participantes não se identificaram diferenças estatísticas para idade $\left(F_{(2,60)}=\right.$ $2,93, p=0,20)$, percentual de gordura $\left(F_{(2,60)}=2,69\right.$, $p=0,24)$ e estado de humor $\left(F_{(2,60)}=2,02, p=0,22\right)$ entre GE e GC antes do início da investigação.

Após receber informação sobre os procedimentos aos quais seriam submetidos, os participantes assinaram um termo de assentimento. Os responsáveis dos atletas assinaram o termo de consentimento livre e esclarecido (TCLE), concordando com os procedimentos metodológicos da investigação. Os procedimentos adotados neste estudo atenderam às normas da Resolução 466/12 do Conselho Nacional de Saúde para pesquisas em seres humanos. O projeto obteve aprovação do Comitê de Ética e Pesquisa em Seres Humanos da Faculdade de Filosofia, Ciências e Letras de Ribeirão Preto (CAE 05166712.8.0000.5407). 


\section{Desenho experimental}

Ambos os grupos (GE e GC) fizeram a mesma planificaçáo de treinamento até a fase do polimento. Somente o GE realizou o polimento (TABELA 1). O polimento teve duraçáo de 3 semanas, adotando-se o método de polimento linear ${ }^{4}$. Assim, foi reduzido somente o volume de treinamento: $80 \%$ para a primeira semana,
$60 \%$ para a segunda e $40 \%$ na terceira semana, seguindo indicações de Mujıka et al. ${ }^{10}$. O GC prolongou o mesociclo competitivo até a data do evento alvo (TABELA 2).

A Escala de Humor de Brunel ${ }^{10}$ (BRUMS) foi preenchida pelos atletas antes do início da temporada, o que foi denominado como pré-teste, e na última semana de cada mesociclo [Geral, Específico, competitivo e polimento (somente para o GE)].

TABELA 1 - Periodização do treinamento para o grupo experimental.

\begin{tabular}{|c|c|c|c|c|c|c|c|c|c|c|c|c|c|c|c|c|c|c|c|c|}
\hline \multirow{2}{*}{$\begin{array}{l}\text { Mesociclo } \\
\text { Microciclo }\end{array}$} & \multicolumn{6}{|c|}{ Geral } & \multicolumn{7}{|c|}{ Específico } & \multicolumn{4}{|c|}{ Competitivo } & \multicolumn{3}{|c|}{ Polimento } \\
\hline & 1 & 2 & 3 & 4 & 5 & 6 & 7 & 8 & 9 & 10 & 11 & 12 & 13 & 14 & 15 & 16 & 17 & 18 & 19 & 20 \\
\hline \multicolumn{21}{|l|}{ Volume (\%) } \\
\hline \multicolumn{21}{|l|}{100} \\
\hline \multicolumn{21}{|l|}{90} \\
\hline \multicolumn{21}{|l|}{80} \\
\hline \multicolumn{21}{|l|}{70} \\
\hline \multicolumn{21}{|l|}{60} \\
\hline \multicolumn{21}{|l|}{50} \\
\hline \multicolumn{21}{|l|}{40} \\
\hline \multicolumn{21}{|l|}{30} \\
\hline \multicolumn{21}{|l|}{20} \\
\hline Intensidade & & & $50-$ & $70 \%$ & & & & & & -85 & & & & & $80-$ & $100 \%$ & & & -100 & \\
\hline
\end{tabular}

TABELA 2 - Periodização do treinamento para o grupo controle.

\begin{tabular}{|c|c|c|c|c|c|c|c|c|c|c|c|c|c|c|c|c|c|c|c|c|}
\hline Mesociclo & \multicolumn{6}{|c|}{ Geral } & \multicolumn{7}{|c|}{ Específico } & \multicolumn{7}{|c|}{ Competitivo } \\
\hline Microciclo & 1 & 2 & 3 & 4 & 5 & 6 & 7 & 8 & 9 & 10 & 11 & 12 & 13 & 14 & 15 & 16 & 17 & 18 & 19 & 20 \\
\hline \multicolumn{21}{|l|}{ Volume (\%) } \\
\hline \multicolumn{21}{|l|}{100} \\
\hline \multicolumn{21}{|l|}{90} \\
\hline \multicolumn{21}{|l|}{80} \\
\hline \multicolumn{21}{|l|}{70} \\
\hline \multicolumn{21}{|l|}{60} \\
\hline \multicolumn{21}{|l|}{50} \\
\hline \multicolumn{21}{|l|}{40} \\
\hline \multicolumn{21}{|l|}{30} \\
\hline \multicolumn{21}{|l|}{20} \\
\hline Intensidade & & & $50-$ & 10 & & & & & & -8 & & & & & & 80 & -10 & $\%$ & & \\
\hline
\end{tabular}




\section{Instrumentos}

O estado de humor foi avaliado pela BRUMS em sua versão validada para atletas adolescentes brasileiros $^{8}$. Embora RoHLFs et al. ${ }^{8}$ não tenham avaliado a consistência interna da BRUMS, para a presente amostra, foi encontrado valor de alpha de Cronbach variando de 0,82 a 0,85 em todos os mesociclos. A BRUMS contém 24 indicadores simples de humor, distribuídos em 6 subescalas: tensão $(T)$, depressão $(\mathrm{D})$, raiva $(\mathrm{R})$, vigor $(\mathrm{V})$, fadiga (F) e confusão mental (CM). Cada subescala contém 4 itens em escala do tipo Likert $(\mathrm{Nada}=0 ;$ Um pouco $=1 ;$ Moderadamente $=2 ;$ Bastante $=3$; Extremamente $=4$ ) e o escore pode variar de 0 a 16 . As subescalas T, D, R, F e CM são consideradas os fatores negativos do humor, sendo o $\mathrm{V}$ considerado o fator positivo. O distúrbio total do humor (DTH) é calculado pela soma dos fatores negativos, subtraindo o escore do fator positivo. Ao resultado final do DTH foi somado 100 para que não houvesse resultados negativos.

A densidade corporal foi determinada por meio da técnica de espessura das dobras cutâneas, por meio de um compasso da marca Lange ${ }^{\oplus}$ (USA), sendo utilizadas as dobras cutâneas triciptal e subescapular, adotando o protocolo de Slaughter et al. ${ }^{11}$, que leva em consideração a etnia (branca ou negra) e a etapa maturacional em função da idade cronológica (púbere - 12 a 14 anos; e pós-púbere 15 a 17 anos) do avaliado. Neste sentido, a etnia foi determinada por meio de autoavaliação. Para as aferiçôes das dobras cutâneas, utilizaram-se as padronizaçóes da International Society for Advancement for Kineanthropometry ${ }^{12}$. O percentual de gordura corporal $(\% \mathrm{G})$ foi determinado por meio da equação de SIRI ${ }^{13}$. Em funçâoo de achados científicos indicarem influência do \%G no $\mathrm{DTH}^{5}$, decidiu-se controlar esta variável nas análises estatísticas.

Dados demográficos (idade, etnia, frequência semanal de treino e horas de treino diária) foram avaliados por intermédio de um questionário construído pelos próprios pesquisadores.

\section{Procedimentos}

A priori, os pesquisadores responsáveis entraram em contato um treinador de uma equipe do estado de Minas Gerais. Os procedimentos, bem como os objetivos do estudo foram devidamente explicados e foi solicitada a autorização para desenvolver a investigação com os seus atletas.

Em seguida, foi realizada uma reunião com os nadadores com o intuito de esclarecer sobre todos os procedimentos éticos da investigação. Neste encontro também foi entregue o TCLE ao seu respectivo treinador para consentimento da participaçáo de seus atletas. Todos os atletas assinaram o termo de assentimento, concordando com a sua participação voluntária na investigação.

As coletas dos dados foram realizadas no local de treinamento (parque aquático). Massa corporal, estatura e dobras cutâneas foram aferidos e em seguida os nadadores responderam a BRUMS antes do primeiro microciclo e ao final de cada mesociclo (último microciclo de cada mesociclo). Os atletas receberam a mesma orientação verbal e eventuais dúvidas foram esclarecidas. Também constavam no questionário orientações escritas sobre o preenchimento do mesmo. A aplicação foi coletiva e respondida de forma individual, tendo duração média de 10 minutos.

\section{Análise dos dados}

O teste Shapiro Wilk não apontou violação paramétrica dos dados da investigação para os dois grupos (GE e GC). Logo, optou-se pela utilização de técnicas paramétricas. Média e desvio-padrão foram utilizados para descrever todas as variáveis (BRUMS e \%G). Conduziu-se a análise multivariada de covariância (MANOVA) de medidas repetidas para comparar as subescalas da BRUMS (T, D, R, F, CM e V) entre os grupos (GE e GC) em função do mesociclo (geral, preparatório, competitivo e polimento). Utilizouse o teste post hoc de Bonferroni para identificar possíveis diferenças estatísticas. Salienta-se que o \%G foi controlado. Ademais, utilizou-se o tamanho do efeito de Cohen, representado pela sigla " $d$ ", para apontar diferenças do ponto de vista prático. Foram adotados os seguintes critérios, de acordo com os apontamentos de Thalheimer e $\operatorname{Cook}^{14}: d<0,4=$ baixo tamanho do efeito, $0,4 \leq d>0,6=$ tamanho do efeito moderado e, $d \geq 0,6=$ grande tamanho do efeito. Todos os dados foram tratados no software SPSS 21.0, adotando-se nível de significância de 5\%. 


\section{Resultados}

A TABELA 3 demonstra a média e o erro padrão das subescalas da BRUMS em função do grupo (GE x GC) e da etapa da periodização do treinamento (geral, específico, competitivo e polimento).

Os resultados apresentaram efeitos de tempo $\left(F_{(4,58)}=34,02, p=0,001\right)$ e grupo $\left(F_{(2,60)}=28,91\right.$, $p=0,001)$ que merecem destaque: a) os achados revelaram que a magnitude da subescala $T$ aumentou progressimente até o polimento tanto no $\operatorname{GE}\left(F_{(4,26)}=45,90, p=0,001\right)$, quanto no GC $\left(F_{(4,28)}=42,83, p=0,001\right)$, não sendo identificada diferença significante entre os grupos $\left(F_{(2,60)}=3,84\right.$, $p=0,18, d=0,1)$ em nenhuma das etapas da periodizaçáo do treinamento; $b$ ) para a subescala $\mathrm{D}$, os resultados indicaram aumento de sua magnitude até o período competitivo em ambos os grupos $\left[\mathrm{GE}\left(F_{(3,27)}=27,83, p=0,01\right), \mathrm{GC}\left(F_{(3,29)}=24,65\right.\right.$, $p=0,02)]$, com atenuação no polimento somente para o GE $\left(F_{(4,26)}=36,81, p=0,01\right)$, havendo difença entre os grupos nesta última etapa $\left(F_{(2,60)}=32,91\right.$, $p=0,001, d=0,5) ; \mathrm{c})$ os achados demonstraram aumento progressivo da subescala $\mathrm{R}$ no $\mathrm{GE}\left(F_{(3,27)}=\right.$ $23,97, p=0,03)$ e GC $\left(F_{(3,29)}=25,81, p=0,02\right)$ até o período competitivo. Em seguida (polimento), ocorreu redução no $\operatorname{GE}\left(F_{(4,26)}=29,72, p=0,01\right)$ e manutenção no $\mathrm{GC}\left(F_{(4,28)}=2,30, p=0,21\right)$, no entanto, foram encontradas diferenças entre os $\operatorname{grupos}\left(F_{(2,60)}=19,84, p=0,01, d=0,5\right)$; d $)$ do mesmo modo, os resultados para a subescala $\mathrm{F}$ apontaram aumento de sua magnitude em ambos os grupos até o período competitivo [GE $\left(F_{(3,27)}=56,89\right.$, $p=0,001)$, GC $\left.\left(F_{(3,29)}=51,45, p=0,001\right)\right]$, todavia, no período seguinte (polimento), acarretou diminuição no GE $\left(F_{(4,26)}=62,33, p=0,001\right) \mathrm{e}$ manutenção no $\mathrm{GC}\left(F_{(4,28)}=2,84, p=0,16\right)$, com diferenças sendo identificadas entre os grupos nesta última etapa $\left(F_{(2,60)}=87,49, p=0,001, d=0,8\right)$; e) em contrapartida, a MANOVA não revelou efeito de tempo $\left(F_{(4,58)}=1,76, p=0,26\right)$ e grupo $\left(F_{(2,60)}=1,95\right.$, $p=0,30, d=0,1)$ para a subescala $\mathrm{CM} ; \mathrm{f})$ o V atenuou progressivamente tanto no $\mathrm{GE}\left(F_{(3,27)}=78,10\right.$, $p=0,001)$ quanto no $\mathrm{GC}\left(F_{(3,27)}=84,39\right.$, $p=0,001)$ até o período competitivo, ao passo que o mesmo no polimento foi aumentado no GE $\left(F_{(4,26)}=92,61, p=0,001\right)$ e mantido no $\mathrm{GC}\left(F_{(4,28)}=2,84, p=0,36\right)$, com diferenças de grupo sendo encontradas para esta última etapa do treinamento $\left(F_{(2,60)}=124,98, p=0,001, d=0,9\right)$.

TABELA 3 - Média e erro padrão dos escores das subescalas da BRUMS em função do grupo e da etapa da periodização do treinamento.

\begin{tabular}{|c|c|c|c|c|c|}
\hline Etapa & & Geral & Específico & Competitivo & Polimento \\
\hline \multirow{2}{*}{ Tensão } & GE & $1,46(0,21)^{\mathrm{b}, \mathrm{c}}$ & $1,56(0,19)^{\mathrm{cdd}}$ & $1,67(0,24)$ & $2,33(0,26)^{a, c}$ \\
\hline & GC & $1,44(0,24)^{\mathrm{b}, \mathrm{c}}$ & $1,58(0,23)^{\mathrm{c}, \mathrm{d}}$ & $1,65(0,20)$ & $2,26(0,23)^{a, c}$ \\
\hline \multirow{2}{*}{ Depressão } & GE & $1,87(0,33)^{\mathrm{c}, \mathrm{d}}$ & $1,82(0,31)^{\mathrm{c}, \mathrm{d}}$ & $1,99(0,28)^{d}$ & $1,51(0,34)^{*}$ \\
\hline & GC & $1,83(0,27)^{\mathrm{c}, \mathrm{d}}$ & $1,79(0,30)^{c, d}$ & $1,94(0,29)$ & $1,96(0,33)$ \\
\hline \multirow{2}{*}{ Raiva } & GE & $1,22(0,36)^{c, d}$ & $1,27(0,38)^{\mathrm{c}, \mathrm{d}}$ & $1,48(0,37)^{\mathrm{d}}$ & $1,10(0,39)^{*}$ \\
\hline & GC & $1,29(0,33)^{\mathrm{c,d}}$ & $1,28(0,41)^{c, d}$ & $1,43(0.35)$ & $1,49(0,39)$ \\
\hline \multirow{2}{*}{ Fadiga } & GE & $1,19(0,43)^{b, c}$ & $1,87(0,38)^{\mathrm{c}, \mathrm{d}}$ & $2,26(0,42)^{d}$ & $1,25(0,41)^{*}$ \\
\hline & $\mathrm{GC}$ & $1,23(0,37)^{\mathrm{b}, \mathrm{c}}$ & $1,80(0,34)^{c, d}$ & $2,17(0,39)$ & $2,23(0,36)^{a}$ \\
\hline \multirow{2}{*}{ Confusão Mental } & GE & $1,05(0,22)$ & $1,08(0,29)$ & $1,11(0,26)$ & $1,09(0,24)$ \\
\hline & GC & $1,02(0,25)$ & $1,07(0,23)$ & $1,13(0,30)$ & $1,14(0,28)$ \\
\hline \multirow[b]{2}{*}{ Vigor } & GE & $12,33(0,47)^{\mathrm{b}, \mathrm{c}}$ & $11,65(0,45)^{c, d}$ & $10,09(0,49)^{\mathrm{d}}$ & $14,92(0,51)^{*}, a$ \\
\hline & $\mathrm{GC}$ & $12,17(0,56)^{b, c}$ & $11,73(0,52)^{\mathrm{c}, \mathrm{d}}$ & $10,38(0,43)$ & $10,21(0,54)^{\mathrm{a}}$ \\
\hline \multirow{2}{*}{ DTH } & GE & $118,40(1,32)^{\mathrm{b}}$ & $120,89(1,24)^{\mathrm{c}, \mathrm{d}}$ & $123,52(1,47)^{\mathrm{a}, \mathrm{d}}$ & $114,62(1,19)^{*}$, a \\
\hline & GC & $117,94(1,21)^{b}$ & $121,08(1,38)^{\mathrm{c}, \mathrm{d}}$ & $123,16(1,29)^{a}$ & $123,75(1,37)^{a}$ \\
\hline
\end{tabular}

$\mathrm{GE}=\mathrm{Grupo}$ experimental; $\mathrm{GC}=$ Grupo controle; $\mathrm{DTH}=$ Distúrbio total do humor; ${ }^{*} p<0,05$ entre os grupos; ${ }^{a} p<0,05$ em relação a etapa "geral"; ${ }^{b} p<0,05$ em relação a etapa "específico"; c $p<0,05$ em relação a etapa "competitivo"; d $p<0,05$ em relação a etapa "polimento". 


\section{Discussão}

A investigação teve como premissa analisar o efeito do polimento sobre o estado de humor de nadadores. Embora revisóes sistemáticas apontem efeito positivo do polimento sobre variáveis fisiológicas ${ }^{4,6}$, pouco se sabe a respeito das adaptações psicológicas oriundas desta etapa da periodizaçáo do treinamento em atletas de esportes cíclicos. De maneira geral, os achados do presente estudo revelaram o aumento do chamado perfil "iceberg" após o polimento para o GE.

Embora o polimento seja uma técnica de prescriçáo do treinamento esportivo considerada consagrada ${ }^{15}$, vale salientar a escassez de investigaçóes científicas concernentes as suas adaptaçóes psicológicas. Pesquisadores salientam que o polimento, caracterizado por redução da carga de treinamento físico, pode acarretar aumento de autoeficácia/autoconfiança, motivação, redução do estresse e melhora do estado de humor dos atletas ${ }^{7}$. Parece que a redução do humor negativo (raiva, depressão e tensão) e o aumento da percepção de vigor estão intimamente relacionados a otimização do desempenho de atletas em competiçóes?.

Os resultados do presente estudo revelaram aumento da $\mathrm{T}$ até o final do macrociclo em ambos os grupos, refutando as hipóteses formuladas inicialmente. Assim, mesmo após a redução da carga de treinamento no GE (polimento), ocorreu aumento da magnitude da $T$, o que pode ser explicado pela proximidade do evento competitivo alvo (campeonato mineiro de inverno). Segundo Mujıka et al. ${ }^{7}$, é comum atletas atingirem níveis elevados de ansiedade em véspera de competição. Cabe destacar que a subescala $T$ da BRUMS assemelha-se aos sintomas de ansiedade. Logo, a $\mathrm{T}$ elevada ao fim das 20 semanas do macrociclo pode indicar o aumento de ansiedade pré-competitiva dos jovens nadadores.

Em relação as demais subescalas negativas do humor (D, R e F), excetuando-se a CM, os achados demonstraram comportamento semelhante. D, R e $\mathrm{F}$ aumentaram em razão da fase da temporada. Ocorreu aumento destas três subescalas até o período competitivo. Este resultado pode ser explicado em virtude do aumento da carga de treinamento ao longo do macrociclo. De acordo com Rouverx et al. ${ }^{\text {, }}$ o humor do atleta pode alterar negativamente após situaçôes que demandam elevado estresse orgânico, mais especificamente, após período de aumento das cargas de treinamento físico. No entanto, D, $\mathrm{R}$ e $\mathrm{F}$ atenuaram no GE na fase de polimento, fato não replicado para o GC. Destaca-se, sobretudo, o elevado tamanho do efeito para a $\mathrm{F}$ quando os grupos foram comparados na etapa do polimento, o que indica grande probabilidade deste efeito ser verdadeiro para a populaçáo de jovens nadadores do sexo masculino. Estudiosos do treinamento esportivo ressaltam que a redução da carga de treinamento gera aumento dos estoques de glicogênio muscular ${ }^{16} \mathrm{e}$ concentração plasmática de serotonina ${ }^{2}$, o que pode explicar, de certo modo, a atenuação da sensação de F, D e R no GE após a fase de polimento.

Os resultados para a subescala $\mathrm{CM}$ não indicaram alteração de sua magnitude em razão da etapa da periodizaçáo para ambos os grupos. Freitas et al. ${ }^{17}$ afirmam que a $\mathrm{CM}$ náo altera durante uma temporada competitiva. Parece que a carga do treinamento não afeta as sensaçóes de insegurança, indecisão e desorientação em atletas. FreITAS et al. ${ }^{17}$ salientam que os níveis de CM são afetados somente quando os atletas também demonstram sintomas clínicos de overtraining.

Em contrapartida, o V sofreu alterações no decorrer de todo o macrociclo. No GE o V atenuou progressivamente até o período competitivo e no polimento demonstrou aumento significante. No GC o $V$ reduziu até o período competitivo e em seguida manteve-se estável. Vale destacar que foi encontrada diferença de V entre GE e GC (elevado tamanho do efeito) somente na etapa de polimento. Pesquisadores afirmam que o polimento é capaz de gerar sensaçóes de energia e disposição aumentadas em atletas ${ }^{7,10}$, o que pode explicar este achado. Estudos indicam estreita relação positiva entre as percepçóes subjetivas de energia/disposição e o desempenho esportivo ${ }^{7,18}$. De acordo com Rohlfs et al. ${ }^{8}$, o V é o aspecto mais importante do humor para atletas que galgam obter sucesso em suas carreiras esportivas. Logo, parece que o polimento com redução progressiva de volume de treinamento, conforme realizado para o GE no presente estudo, pode ser recomendado para a melhora do $\mathrm{V}$ e possível consequencia positiva no desempenho em competição.

Embora a presente investigação seja dotada de achados inéditos e importantes, a mesma demonstra limitaçóes que merecem destaque. Utilizou-se questionário como instrumento de avaliação. Logo, as respostas podem não refletir a realidade do contexto avaliado ${ }^{19,20}$. Aponta-se também o uso de método duplamente indireto (dobras cutâneas) para se avaliar o percentual de gordura como limitação. 
A despeito destas limitaçóes, pressupôe-se que esta pesquisa revele resultados interessantes que merecem ser discutidos na literatura científica da área das ciências do esporte.

Poucos estudos têm sido desenvolvidos em prol de se investigar os efeitos de técnicas de prescrição do treinamento nos esportes sobre variáveis que estão direta ou indiretamente relacionadas ao desempenho. Mais especificamente, embora o polimento seja mundialmente conhecido, raríssimas pesquisas foram conduzidas com o intuito de se avaliar os seus efeitos em variáveis psicológicas.

Em suma, os resultados do presente estudo permitiram concluir que o polimento foi eficaz para melhorar o estado de humor de nadadores, com destaque para o aumento do $\mathrm{V}$ e diminuição da $\mathrm{R}$, F e D. Todavia, estes achados devem ser analisados com cautela em razão de se tratarem de respostas subjetivas. Do ponto de vista prático, considerando que o aumento do vigor e a redução da sensação de fadiga são encontrados em atletas que obtiveram sucesso em competiçôes ${ }^{7,18}$, pode-se presumir que a etapa do polimento é essencial para potencializar as capacidades mentais e, consequentemente, maximizar o desempenho esportivo. Por fim, sugerese a condução de mais investigaçóes para confirmar tais resultados. Ademais, recomenda-se que pesquisas sejam realizadas com atletas do sexo feminino.

\section{Abstract}

\section{Effect of tapering on mood in swimmers}

There seems to be a positive relationship between mood and sport performance. However, although researchers claim that tapering can result in improved mood, it is worth noting that such adaptation needs to be confirmed from scientific findings. Little is known about the effect of tapering on mood of athletes. The objective was to analyze the effect of tapering on the mood in swimmers. 62 male swimmers were participants, randomly divided into experimental (EG) and control (CG) group. Both groups did the same planning training to the tapering phase. Only EG has made tapering. Tapering lasted three weeks, adopting the linear tapering method. The Brunel Mood Scale (BRUMS) was filled by athletes before the start of the season and the last week of each mesocycle. It led to covariance multivariate analysis (MANOVA) of repeated measures to compare the BRUMS subscales between groups according to mesocycle. The results showed an effect of time $\left(F_{(4,58)}=34,02, p=0,001\right)$ and group $\left(F_{(2,60)}=28,91, p=0,001\right)$. Overall, the findings of this study revealed the increased profile called "iceberg" after tapering for EG. It was concluded that the tapering was effective to improve the mood of swimmers, especially the increase in the subjective force and decreased anger, fatigue and depression.

KeYwords: Sport Psychology; Mood; Athletes.

\section{Agradecimentos}

Aos atletas pela colaboração em participar da investigação. Ao Banco Santander pelo financiamento de parte do projeto.

\section{Referências}

1. Ronnestad BR, Hansen J, Ellefsen HS. Block periodization of high-intensity aerobic intervals provides superior training effects in trained cyclists. Scand J of Med Sci Sports. 2014;24(1):34-42.

2. Pyne DB, Mujika I, Reilly T. Peaking for optimal performance: research limitations and future directions. J Sports Sci. 2009;27(3):195-202. 
3. Hellard P, Avalos M, Hausswirth C, Pyne D, Toussaint J, Mujika I. Identifying optimal overload and taper in elite swimmers over time. J Sports Sci Med. 2013;12(4):668-78.

4. Bosquet L, Montpetit J, Arvisais D, Mujia I. Effects of tapering on performance: a meta-analysis. Med Sci Sports Exercise. 2007;39(8):1358-65.

5. Fortes LS, Almeida SS, Ferreira MEC. Influência da periodizaçáo do treinamento sobre os comportamentos de risco para transtornos alimentares em nadadoras. Rev Educ Fís UEM. 2014;25(1):127-34.

6. le Meur Y, Hausswirth C, Mujika I. Tapering for competition: a review. Sci Sports. 2012;27(2):77-87.

7. Mujika I, Padilla S, Pyne D. Swimming performance changes during the final 3 weeks of training leading to the Sydney 2000 Olympic Games. Int J Sports Med. 2002;23(8):582-7.

8. Rohlfs ICPM, Rotta TM, Luft CDB, Andrade A, Krebs RJ, Carvalho T. A escala de humor de Brunel (Brums): instrumento para detecção precoce da síndrome do excesso de treinamento. Rev Bras Med Esporte. 2008;14(3):176-81.

9. Rouveix M, Bouget M, Pannafieux C, Champely S, Filaire E. Eating attitudes, body esteem, perfectionism and anxiety of judo athletes and nonathletes. Int J Sports Med. 2007;28(4):340-5.

10. Mujika I, Chaouachi A, Chamari K. Precompetition taper and nutritional strategies: special reference to training during Ramadan intermittent fast. Br J Sports Med. 2010;44(7):495-501.

11. Slaughter MH, Lohman TG, Boileau R, Hoswill CA, Stillman RJ, Yanloan MD, et al. Skinfold equations for estimation of body fatness in children and youth. Hum Biol. 1988;60(3):709-23.

12. The International Society for Advancement for Kineanthropometry [homepage na Internet]. Sidney: National Library of Australia; [data desconhecida] [citado em 2013 jul 3]. Disponível em: <https://goo.gl/sN34Ug>.

13. Siri WE. The gross composition of the body. In: Tobias CA, Lawrence JH, editores. Advances in biological and medical physics. New York: Academic Press; 1956. p. 239-80.

14. Thalheimer W, Cook S. How to calculate effect sizes from published research articles: a simplified methodology. Somerville: Work-Learning Research; 2002.

15. Mujika I. Intense training: the key to optimal performance before and during the taper. Scand J Med Sci Sports. 2010;20(supl 2):24-31.

16. Spriet LL. New insights into the interaction of carbohydrate and fat metabolism during exercise. Sports Med. 2014; 44(supl 1):87-96.

17. Freitas DS, Miranda R, Bara Filho MG. Marcadores psicológico, fisiológico e bioquímico para determinação dos efeitos da carga de treino e do overtraining. Rev Bras Cineantropom Desempenho Hum. 2009;11(4):457-65.

18. Vieira LF, Oliveira JS, Gaion PA, Oliveira HG, Rocha PGM, Vieira JLL. Mood states and training: a study with high performance deep runners athletes. Rev Educ Fís UEM. 2010;21(4):585-91.

19. Fortes LS, Ferreira MEC. Comparison of body dissatisfaction and inappropriate eating behavior in adolescent athletes of different sports. Rev Bras Educ Fís Esporte. 2011;25(4):707-16.

20. Fortes LS, Matta MO, Paes ST, Ferreira MEC. Fatores de risco associados ao comportamento alimentar inadequado em futebolistas. Rev Bras Educação Fís Esporte. 2012;26(3):447-54.

\begin{tabular}{|c|c|}
\hline $\begin{array}{r}\text { ENDEREÇO } \\
\text { Leonardo de Sousa Fortes } \\
\text { Rua Clóvis Beviláqua, 163/1.003 - Madalena } \\
\text { 50710-330 - Recife - PE - BRASIL } \\
\text { e-mail: leodesousafortes@hotmail.com }\end{array}$ & 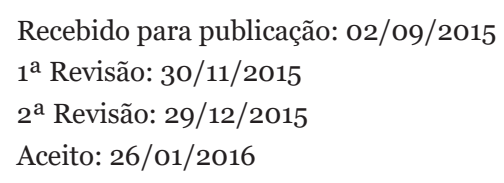 \\
\hline
\end{tabular}

